\title{
Multiobjective Duality in Variational Problems with Higher Order Derivatives
}

\author{
Iqbal Husain ${ }^{1}$, Rumana G. Mattoo ${ }^{2}$ \\ ${ }^{1}$ Department of Mathematics, Jaypee Institute of Engineering and Technology, Guna, India \\ ${ }^{2}$ Department of Statistics, University of Kashmir, Srinagar, India \\ E-mail: \{ihusain11,rumana_research\}@yahoo.com \\ Received December 16, 2009; revised April 25, 2010; accepted April 30, 2010
}

\begin{abstract}
A multiobjective variational problem involving higher order derivatives is considered and optimality conditions for this problem are derived. A Mond-Weir type dual to this problem is constructed and various duality results are validated under generalized invexity. Some special cases are mentioned and it is also pointed out that our results can be considered as a dynamic generalization of the already existing results in nonlinear programming.
\end{abstract}

Keywords: Multiobjective Variational Problem; Efficiency, Duality, Pseudoinvexity, Quasinvexity, Nonlinear Programming

\section{Introduction}

Calculus of variation is a powerful technique for the solution of various problems appearing in dynamics of rigid bodies, optimization of orbits, theory of variations and many other fields. The subjects whose importance is fast growing in science and engineering primarily concern with finding optimal of a definite integral involving a certain function subject to fixed point boundary conditions. In [1] Courant and Hilbert, quoting an earlier work of Friedrichs [2], gave a dual relationship for a simple type of unconstrained variational problem. Subsequently, Hanson [3] pointed out that some of the duality results of mathematical programming have analogous in variational calculus. Exploring this relationship between mathematical programming and the classical calculus of variations, Mond and Hanson [4] formulated a constrained variational problem as a mathematical programming problem and using Valentine's [5] optimality conditions for the same, presented its Wolfe type dual variational problem for validating various duality results under convexity. Later Bector, Chandra and Husain [6] studied Mond-Weir type duality for the problem of [4] for weakening its convexity requirement. In [7] Chandra, Craven and Husain studied optimality and duality for a class of non-differentiable variational problem with non-differentiable term in the integrand of the objective functional while in [8] they derived optimality conditions and duality results for a constrained variational problem having terms with arbitrary norms in the objective as well as constrained functions.

Recently Husain and Jabeen [9] studied a wider class of variational problem in which the arc function is twice differentiable by extending the notion of invexity given in [10]. They obtained Fritz John as well as KarushKuhn-Tucker necessary optimality conditions as an application of Karush-Kuhn-Tucker optimality conditions studied various duality results for Wolfe and Mond and Weir type models.

In single objective programming we must settle on a single objective such as minimizing cost or maximizing profit. However, generally any real world problems can be identified with multiple conflicting criteria e.g., the problems of oil refinery scheduling, production planning, portfolio selection and many others can be modelled as multiobjective programming problems.

Duality results are very useful in the development of numerical algorithms for solving certain classes of optimization problems. Duality for multiobjective variational problem has been studied by a number of authors, notably Bector and Husain [11], Chen [12] and many others cited in these references. Applications of duality theory are prominent in physics, economics, management sciences, etc.

Since mathematical programming and classical calculus of variations have undergone independent development, it is felt that mutual adaptation of ideas and tech- 
niques may prove useful. Motivated with this idea in this exposition, we propose to study optimality criteria and duality for a wider class of multiobjective variational problems involving higher order derivative. These results not only generalize the results of Husain and Jabeen [9] and Bector and Husain [11] but also present a dynamic generalization of some of the results in multiobjective nonlinear programming already existing.

\section{Invexity and Generalized Invexity}

Invexity was introduced for functions in variational problems by Mond, Chandra and Husain [10] while Mond and Smart [13] defined invexity for functionals instead of functions. Here we introduce extended forms of definitions of invexity and various generalized invexity for functional in variational problems involving higher order derivatives.

Consider the real interval $I=[a, b]$, and the continuously differentiable function $\phi: I \times R^{n} \times R^{n} \times R^{n} \rightarrow R$, where $x$ is twice differentiable with its first and second order derivatives $\dot{x}$ and $\ddot{x}$ respectively. If $x=\left(x^{1}, x^{2}, \ldots, x^{n}\right)^{T}$, the gradient vectors of $f$ with respect to $x, \dot{x}$ and $\ddot{x}$ respectively denoted by

$$
\begin{gathered}
\phi_{x}=\left[\frac{\partial \phi}{\partial x^{1}}, \ldots, \frac{\partial \phi}{\partial x^{n}}\right]^{T}, \phi_{\dot{x}}=\left[\frac{\partial \phi}{\partial \dot{x}^{1}}, \ldots, \frac{\partial \phi}{\partial \dot{x}^{n}}\right]^{T} \\
\phi_{\ddot{x}}=\left[\frac{\partial \phi}{\partial \ddot{x}^{1}}, \ldots, \frac{\partial \phi}{\partial \ddot{x}^{n}}\right]^{T} .
\end{gathered}
$$

DEFINITION 1. (Invexity): If there exists vector function $\quad \eta(t, \dot{u}, \ddot{u}, x, \dot{x}, \ddot{x}) \in R^{n} \quad$ with $\quad \eta=0 \quad$ and $x(t)=u(t), t \in I \quad$ and $D \eta=0 \quad$ for $\quad \dot{x}(t)=\dot{u}(t), t \in I$ such that for a scalar function $\phi(t, x, \dot{x}, \ddot{x})$, the functional $\Phi(x, \dot{x}, \ddot{x})=\int_{I} \phi(t, x, \dot{x}, \ddot{x}) d t$ satisfies

$$
\begin{gathered}
\Phi(x, \dot{u}, \ddot{u})-\Phi(x, \dot{x}, \ddot{x}) \geq \\
\int_{I}\left\{\eta^{T} \phi_{x}(t, x, \dot{x}, \ddot{x})+(D \eta)^{T} \phi_{\dot{x}}(t, x, \dot{x}, \ddot{x})+\right. \\
\left.\left(D^{2} \eta\right)^{T} \phi_{\ddot{x}}(t, x, \dot{x}, \ddot{x})\right\} d t
\end{gathered}
$$

$\Phi$ is said to be invex in $x, \dot{x}$ and $\ddot{x}$ on $I$ with respect to $\eta$.

Here $D$ is a differentiation operator defined later.

DEFINITION 2. (Pseudoinvexity): $\Phi$ is said to be pseudoinvex in $x, \dot{x}$ and $\ddot{x}$ with respect to $\eta$ if

$$
\int_{I}\left\{\eta^{T} \phi_{x}(t, x, \dot{x}, \ddot{x})+(D \eta)^{T} \phi_{\dot{x}}(t, x, \dot{x}, \ddot{x})\right.
$$

$$
\left.+\left(D^{2} \eta\right)^{T} \phi_{\ddot{x}}(t, x, \dot{x}, \ddot{x})\right\} d t \underset{=}{=}
$$

implies $\Phi(x, \dot{u}, \ddot{u}) \geq \Phi(x, \dot{x}, \ddot{x})$.

DEFINITION 3. (Quasi-invex): The functional $\Phi$ is said to quasi-invex in $x, \dot{x}$ and $\ddot{x}$ with respect to $\eta$ if $\Phi(x, \dot{u}, \ddot{u}) \leq \Phi(x, \dot{x}, \ddot{x})$ implies

$$
\begin{gathered}
\int_{I}\left\{\eta^{T} \phi_{x}(t, x, \dot{x}, \ddot{x})+(D \eta)^{T} \phi_{\dot{x}}(t, x, \dot{x}, \ddot{x})+\right. \\
\left.+\left(D^{2} \eta\right)^{T} \phi_{\ddot{x}}(t, x, \dot{x}, \ddot{x})\right\} d t \lesseqgtr 0
\end{gathered}
$$

\section{Variational Problem and Optimality Conditions}

Before stating our variational problem and deriving its necessary optimality condition, we mention the following conventions for vectors $x$ and $y$ in $n$-dimensional Euclidian space $R^{n}$ will be used throughout the analysis of this research.

$$
\begin{aligned}
& x<y, \quad \Leftrightarrow \quad x_{i}<y_{i}, \quad i=1,2, \ldots, n . \quad x \\
& x \leq y, \quad \Leftrightarrow \quad x_{i} \leq y_{i}, \quad i=1,2, \ldots, n . \\
& x \leq y, \quad \Leftrightarrow \quad x_{i} \leq y_{i}, \quad i=1,2, \ldots, n, \text { but } x \neq y \\
& x \not z y \text {, is the negation of } x \leq y
\end{aligned}
$$

For $x, y \in R, x \leq y$ and $x<y$ have the usual meaning.

In this section, we present the following variational problem whose optimality conditions will be derived and duality will be investigated in the subsequent sections:

(VPE) Minimize

$$
\left(\int_{I} f^{1}(t, x, \dot{x}, \ddot{x}) d t, \ldots, \int_{I} f^{p}(t, x, \dot{x}, \ddot{x}) d t\right)
$$

Subject to

$$
\begin{gathered}
x(a)=0=x(b) \\
\dot{x}(a)=0=\dot{x}(b) \\
g(t, x, \dot{x}, \ddot{x}) \leq 0 \quad, \quad t \in I \\
h(t, x, \dot{x}, \ddot{x})=0, \quad t \in I
\end{gathered}
$$

where $f^{i}: I \times R^{n} \times R^{n} \times R^{n} \rightarrow R, i=1,2, \ldots, p$, $g: I \times R^{n} \times R^{n} \times R^{n} \rightarrow R^{m}$ and $h: I \times R^{n} \times R^{n} \times R^{n} \rightarrow R^{k}$ are continuously differentiable functions, and $\mathrm{X}$ designates the space of piecewise functions $x: I \rightarrow R^{n}$ possessing derivatives $\dot{x}$ and $\ddot{x}$ with the norm $\|x\|=\|x\|_{\infty}+$ $\|D x\|_{\infty}+\left\|D^{2} x\right\|_{\infty}$, where the differentiation operator $D$ is given by 


$$
u=D x \Leftrightarrow x(t)=\alpha+\int_{a}^{t} u(s) d s
$$

where $\alpha$ is given boundary value; thus $D \equiv \frac{d}{d t}$ except at discontinuities.

In the results to follow, we use $C\left(I, R^{m}\right)$ to denote the space of continuous functions $\phi: I \rightarrow R^{k}$ with the uniform norm $\|\phi\|=\sup |\phi|_{t \in I}$; the partial derivatives of $g$ and $h$ are $m \times n$ and $k \times n$ matrices respectively; superscript $T$ denotes matrix transpose.

We require the following definition of efficient solution for our further analysis.

DEFINITION 4. (Efficient Solution): A feasible solution $\bar{x}$ is efficient for (VPE) if there exist no other feasible $x$ for (VPE) such that for some $i \in P=\{1,2, \ldots, p\}$,

$$
\int_{I} f^{i}(t, x, \dot{x}, \ddot{x}) d t<\int_{I} f^{i}(t, \bar{x}, \dot{\bar{x}}, \ddot{\bar{x}}) d t
$$

and $\int_{I} f^{i}(t, x, \dot{x}, \ddot{x}) d t \leq \int_{I} f^{i}(t, \bar{x}, \dot{\bar{x}}, \ddot{\bar{x}}) d t \quad$ for all $j \in P$, $j \neq i$.

In relation to (VPE), we introduce the following set of problems $\bar{P}_{r}$ for each $r=1,2, \ldots, p$ in the spirit of [14], with a single objective,

$$
\left(\bar{P}_{r}\right) \text { Minimize } \int_{I} f^{r}(t, x, \dot{x}, \ddot{x}) d t
$$

Subject to

$$
\begin{gathered}
x(a)=0=x(b), \\
\dot{x}(a)=0=\dot{x}(b), \\
g(t, x, \dot{x}, \ddot{x}) \leq 0, t \in I, \\
h(t, x, \dot{x}, \ddot{x})=0, t \in I, \\
\int_{I} f^{i}(t, x, \dot{x}, \ddot{x}) d t \leq \int_{I} f^{i}(t, \bar{x}, \dot{\bar{x}}, \ddot{\bar{x}}) d t, i=1,2, \ldots, p, i \neq r
\end{gathered}
$$

The following lemma can be proved on the lines of Chankong and Haimes [14].

LEMMA 1: $x^{*}$ is an efficient solution of (VPE) if and only if $\bar{x}$ is an optimal solution of $\left(\bar{P}_{r}\right)$ for each $r=1,2, \ldots, p$.

$\left(\mathbf{P}_{\mathbf{0}}\right)$ Minimize $\int_{I} \phi(t, x, \dot{x}, \ddot{x}) d t$

Subject to

$$
\begin{gathered}
x(a)=0=x(b), \\
\dot{x}(a)=0=\dot{x}(b), \\
g(t, x, \dot{x}, \ddot{x}) \leq 0, t \in I,
\end{gathered}
$$

$$
h(t, x, \dot{x}, \ddot{x})=0 \quad, \quad t \in I,
$$

where $\phi: I \times R^{n} \times R^{n} \times R^{n} \rightarrow R$.

PROPOSITION 1. [9]: (Fritz John Optimality Conditions) If $\bar{x}$ is an optimal solution of $\left(\mathrm{P}_{0}\right)$ and $h_{x}(x(\cdot), \dot{x}(\cdot), \ddot{x}(\cdot))$ maps $\mathrm{X}$ into the subspace of $C\left(I, R^{k}\right)$, then there exists Lagrange multiplier $\bar{\tau} \in R$, the piecewise smooth $\bar{y}: I \rightarrow R^{m}$ and $\bar{z}: I \rightarrow R^{k}$, such that

$$
\begin{gathered}
\left(\bar{\tau} \phi_{x}+\bar{y}(t)^{T} g_{x}+\bar{z}(t)^{T} h_{x}\right)- \\
-D\left(\bar{\tau} \phi_{\dot{x}}+\bar{y}(t)^{T} g_{\dot{x}}+\bar{z}(t)^{T} h_{\dot{x}}\right) \\
+D^{2}\left(\bar{\tau} \phi_{\dot{x}}+\bar{y}(t)^{T} g_{\ddot{x}}+\bar{z}(t)^{T} h_{\ddot{x}}\right)=0, t \in I \\
\bar{y}(t)^{T} g(t, \bar{x}, \dot{\bar{x}}, \ddot{\bar{x}})=0, t \in I, \\
(\bar{\tau}, \bar{y}(t)) \geq 0, t \in I, \\
(\bar{\tau}, \bar{y}(t), \bar{z}(t)) \neq 0, t \in I .
\end{gathered}
$$

If $\bar{\tau}=1$, then the above optimality conditions will reduce to the Karush-Kuhn-Tucker type optimality conditions and the solution $\bar{x}$ is referred to as a normal solution.

We now establish the following theorem that gives the necessary optimality conditions for (VPE).

THEOREM 1: (Fritz-John Conditions): Let $\bar{x}$ be an efficient solution of (VPE) and $h_{x}(x(\cdot), \dot{x}(\cdot), \ddot{x}(\cdot))$ maps $\mathrm{X}$ into the subspace of $C\left(I, R^{k}\right)$, then there exist $\bar{\lambda} \in R^{k}$ and the piecewise smooth $\bar{y}: I \rightarrow R^{m}$ and $\bar{z}: I \rightarrow R^{k}$, such that

$$
\begin{gathered}
\left(\bar{\lambda}^{T} f_{x}+\bar{y}(t)^{T} g_{x}+\bar{z}(t)^{T} h_{x}\right)-D\left(\bar{\lambda}^{T} f_{\dot{x}}+\bar{y}(t)^{T} g_{\dot{x}}+\bar{z}(t)^{T} h_{\dot{x}}\right) \\
+D^{2}\left(\bar{\lambda}^{T} f_{\ddot{x}}+\bar{y}(t)^{T} g_{\ddot{x}}+\bar{z}(t)^{T} h_{\ddot{x}}\right)=0, t \in I \\
\bar{y}(t)^{T} g(t, \bar{x}, \dot{\bar{x}}, \ddot{\bar{x}})=0, t \in I \\
(\bar{\lambda}, \bar{y}(t)) \geq 0, t \in I \\
(\lambda, \bar{y}(t), \bar{z}(t)) \neq 0, t \in I
\end{gathered}
$$

PROOF: Since $\bar{x}$ is an efficient solution of (VPE) by Lemma $1, \bar{x}$ is an optimal solution of $\left(\bar{P}_{r}\right)$, for each $r=1,2, \ldots, p$. From Proposition1, it follows that, there exist scalars $\bar{\lambda}^{1 r}, \bar{\lambda}^{2 r}, \ldots, \bar{\lambda}^{p r}$ and piecewise smooth function $\bar{y}: I \rightarrow R^{m}$ and $\bar{z}: I \rightarrow R^{k}$, such that 


$$
\begin{aligned}
& \bar{\lambda}^{r r} f_{x}^{r}+\sum_{\substack{i=1 \\
i \neq r}}^{p} \bar{\lambda}^{i r} f_{x}^{i}+\sum_{j=1}^{m} \bar{y}^{j r}(t) g_{x}^{j}+\sum_{l=1}^{k} \bar{z}^{l r}(t) h_{x}^{l} \\
& -D\left(\bar{\lambda}^{r r} f_{\dot{x}}^{r}+\sum_{\substack{i=1 \\
i \neq r}}^{p} \bar{\lambda}^{i r} f_{\dot{x}}^{i}+\sum_{j=1}^{m} \bar{y}^{j r}(t) g_{\dot{x}}^{j}+\sum_{l=1}^{k} \bar{z}^{l r}(t) h_{\dot{x}}^{l}\right) \\
& +D^{2}\left(\bar{\lambda}^{r r} f_{\ddot{x}}^{r}+\sum_{\substack{i=1 \\
i \neq r}}^{p} \bar{\lambda}^{i r} f_{\ddot{x}}^{i}+\sum_{j=1}^{m} \bar{y}^{j r}(t) g_{\ddot{x}}^{j}+\sum_{l=1}^{k} \bar{z}^{l r}(t) h_{\ddot{x}}^{l}\right)=0, \\
& t \in I, \\
& \bar{y}^{T}(t) g(t, \bar{x}, \dot{\bar{x}}, \ddot{\bar{x}})=0, t \in I \text {, } \\
& \left(\bar{\lambda}^{1 r}, \bar{\lambda}^{2 r}, \ldots, \bar{\lambda}^{p r}, \bar{y}^{1 r}(t), \bar{y}^{2 r}(t), \ldots, \bar{y}^{m r}(t)\right) \geq 0, t \in I \\
& \left(\bar{\lambda}^{1 r}, \bar{\lambda}^{2 r}, \ldots, \bar{\lambda}^{p r}, \bar{y}^{1 r}(t), \bar{y}^{2 r}(t), \ldots, \bar{y}^{m r}(t), \bar{z}^{1 r}(t), \bar{z}^{2 r}(t),\right. \\
& \left.\ldots, \bar{z}^{l r}(t)\right) \neq 0, t \in I \text {. }
\end{aligned}
$$

Summing over $r$, we have

$$
\begin{gathered}
\sum_{r=1}^{p}\left(\sum_{i=1}^{p} \bar{\lambda}^{i r}\right) f_{x}^{i}+\sum_{r=1}^{p}\left(\sum_{j=1}^{m} \bar{y}^{j r}(t)\right) g_{x}^{j}+\sum_{r=1}^{p}\left(\sum_{l=1}^{k} \bar{z}^{l r}(t)\right) h_{x}^{l} \\
-D\left(\sum_{r=1}^{p}\left(\sum_{i=1}^{p} \bar{\lambda}^{i r}\right) f_{\dot{x}}^{i}+\sum_{r=1}^{p}\left(\sum_{j=1}^{m} \bar{y}^{j r}(t)\right) g_{\dot{x}}^{j}+\sum_{r=1}^{p}\left(\sum_{l=1}^{k} \bar{z}^{l r}(t)\right) h_{\dot{x}}^{l}\right) \\
+D^{2}\left(\sum_{r=1}^{p}\left(\sum_{i=1}^{p} \bar{\lambda}^{i r}\right) f_{\ddot{x}}^{i}+\sum_{r=1}^{p}\left(\sum_{j=1}^{m} \bar{y}^{j r}\right) g_{\ddot{x}}^{j}+\sum_{r=1}^{p}\left(\sum_{l=1}^{k} \bar{z}^{l r}(t)\right) h_{\ddot{x}}^{l}\right) \\
=0, t \in I \\
\left(\sum_{r=1}^{p} \bar{\lambda}^{1 r}, \ldots, \sum_{i=1}^{p} \bar{\lambda}^{p r} ; \sum_{i=1}^{p} \bar{y}^{1 r}(t), \ldots, \sum_{i=1}^{p} \bar{y}^{m r}(t)\right) \geq 0, t \in I \\
\left(\sum_{r=1}^{p} \bar{\lambda}^{1 r}, \ldots, \sum_{i=1}^{p} \bar{\lambda}^{p r} ; \sum_{i=1}^{p} \bar{y}^{1 r}(t), \ldots, \sum_{i=1}^{p} \bar{y}^{m r}(t) ; \sum_{r=1}^{p} \bar{z}^{1 r}(t),\right. \\
\left.\ldots, \sum_{r=1}^{p} \bar{z}^{l r}(t)\right) \neq 0, t \in I .
\end{gathered}
$$

Setting $\bar{\lambda}^{i}=\sum_{r=1}^{p} \bar{\lambda}^{i r}, \bar{y}^{j}(t)=\sum_{r=1}^{p} \bar{y}^{j r}(t), t \in I \quad$ and $\bar{z}^{l}(t)=\sum_{r=1}^{l} \bar{z}^{l r}(t), t \in I$, we have

$$
\begin{gathered}
\left(\bar{\lambda}^{T} f_{x}+\bar{y}(t)^{T} g_{x}+\bar{z}(t)^{T} h_{x}\right)-D\left(\bar{\lambda}^{T} f_{\dot{x}}+\bar{y}(t)^{T} g_{\dot{x}}+\bar{z}(t)^{T} h_{\dot{x}}\right) \\
+D^{2}\left(\bar{\lambda}^{T} f_{\ddot{x}}+\bar{y}(t)^{T} g_{\ddot{x}}+\bar{z}(t)^{T} h_{\ddot{x}}\right)=0, t \in I
\end{gathered}
$$

$$
\begin{gathered}
\bar{y}(t)^{T} g(t, \bar{x}, \dot{\bar{x}}, \ddot{\bar{x}})=0, t \in I, \\
(\bar{\lambda}, \bar{y}(t)) \stackrel{\geq}{=}, t \in I, \\
(\bar{\lambda}, \bar{y}(t), \bar{z}(t)) \neq 0, t \in I .
\end{gathered}
$$

\section{Mond-Weir Type Duality}

In this section, we consider the following variational problem involving higher order derivatives, by suppressing the equality constraint in (VPE).

(VP) Minimize

$$
\left(\int_{I} f^{1}(t, x, \dot{x}, \ddot{x}) d t, \ldots, \int_{I} f^{p}(t, x, \dot{x}, \ddot{x}) d t\right)
$$

Subject to

$$
\begin{gathered}
x(a)=0=x(b) \\
\dot{x}(a)=0=\dot{x}(b) \\
g(t, x, \dot{x}, \ddot{x}) \leq 0 \quad, \quad t \in I
\end{gathered}
$$

We formulate the following Mond-Weir type dual to the problem (VP) and establish various duality results under invexity defined in the preceding section.

(M-WD) Maximize

$$
\int_{I} f^{1}(t, u, \dot{u}, \ddot{u}) d t, \ldots, \int_{I} f^{p}(t, u, \dot{u}, \ddot{u}) d t
$$

Subject to

$$
\begin{gathered}
x(a)=0=x(b) \\
\dot{x}(a)=0=\dot{x}(b) \\
\left(\lambda^{T} f_{x}+y(t)^{T} g_{x}\right)-D\left(\lambda^{T} f_{\dot{x}}+y(t)^{T} g_{\dot{x}}\right) \\
+D^{2}\left(\lambda^{T} f_{\ddot{x}}+y(t)^{T} g_{\ddot{x}}\right)=0 \\
\int_{1} y(t)^{T} g(t, u, \dot{u}, \ddot{u}) d t \geq 0 \\
y(t) \stackrel{\geq}{=}, \quad t \in I \\
\lambda>0 .
\end{gathered}
$$

THEOREM 2. (Weak Duality): Let $x \in X$ be feasible for (VP) and $(u, \lambda, y)$ be feasible for (M-WD) if for allfeasible $(x, u, \lambda, y) \int_{I} \lambda^{T} f(t, u, \dot{u}, \ddot{u}) d t$ is pseudoinvex and $\int_{I} y(t)^{T} g(t, u, \dot{u}, \ddot{u}) d t$ is quasi-invex with respect to the same $\eta$.

Then, 


$$
\int_{I} f(t, x, \dot{x}, \ddot{x}) d t \not \subset \int_{I} f(t, u, \dot{u}, \ddot{u}) d t .
$$

PROOF: The relations $g(t, x, \dot{x}, \ddot{x}) \leqq 0, \quad y(t) \geqq 0$, $t \in I$ imply

$$
\int_{I} y(t)^{T} g(t, x, \dot{x}, \ddot{x}) d t \leqq \int_{I} y(t)^{T} g(t, u, \dot{u}, \ddot{u}) d t
$$

This, because of the quasi-invexity of $\int_{I} y(t)^{T} g(t, u, \dot{u}, \ddot{u}) d t$, implies that

$$
\begin{aligned}
0 \geq \int_{I} & \left\{\eta^{T} y^{T}(t) g_{u}+(D \eta)^{T} y^{T} g_{u}+\left(D^{2} \eta\right)^{T} y^{T} g_{u i}\right\} d t \\
& =\int_{I} \eta^{T} y(t)^{T} g_{u} d t+\left.\eta^{T} y(t)^{T} g_{u}\right|_{t=a} ^{t=b} \\
& -\int_{I} \eta^{T} D y(t)^{T} g_{\dot{u}} d t+(D \eta)^{T} y(t)^{T} g_{u}||_{t=a}^{t=b} \\
& -\int_{I}(D \eta)^{T} D y(t)^{T} g_{u} d t
\end{aligned}
$$

(By integration by parts)

Using the boundary conditions which gives

$D \eta=0=\eta$ at $t=a, t=b$

$$
\begin{aligned}
& =\int_{I} \eta^{T} y(t)^{T} g_{u} d t-\int_{I} \eta^{T} D y(t)^{T} g_{\dot{u}} d t \\
& -\left.\eta^{T} D y(t)^{T} g_{u ̈}\right|_{t=a} ^{t=b}+\int_{I} \eta^{T} D^{2} y(t)^{T} g_{u} d t
\end{aligned}
$$

(By integration by parts)

Using the boundary conditions which give $D \eta=0=\eta$ at $t=a, t=b$

$$
\begin{gathered}
\int_{I} \eta^{T} y(t)^{T} g_{u} d t-\int_{I} \eta^{T} D y(t)^{T} g_{u} d t+\int_{I} \eta^{T} D^{2} y(t)^{T} \stackrel{g_{u}}{ } d t \leq 0 \\
\int_{I} \eta^{T}\left(y(t)^{T} g_{u}-D y(t)^{T} g_{u}+D^{2} y(t)^{T} g_{u}\right) d t \stackrel{=}{=}
\end{gathered}
$$

From Equation (11) this yields,

$$
\int_{I} \eta^{T}\left\{\lambda^{T} f_{x}-D \lambda^{T} f_{\dot{x}}+D^{2} \lambda^{T} f_{\dot{x}}\right\} d t>0
$$

This by integration by parts and then using boundary conditions gives,

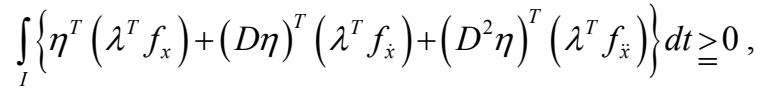

This, in view of psedoinvexity of $\int_{I} \lambda^{T} f(t, x, \dot{x}, \ddot{x}) d t$ implies that

$$
\lambda^{T} \int_{I} f(t, x, \dot{x}, \ddot{x}) d t \geq \lambda^{T} \int_{I} f(t, u, \dot{u}, \ddot{u}) d t .
$$

For this, it follows

$$
\int_{I} f(t, x, \dot{x}, \ddot{x}) d t \not \subset \int_{I} f(t, u, \dot{u}, \ddot{u}) d t .
$$

THEOREM 3. (Strong Duality): If $\bar{x}$ is a feasible solution for (VP) and assume that $\bar{x}$ is an efficient solution and for at least one $i, i \in P, \bar{x}$ satisfies a regularity condition for [7] for $\mathrm{P}_{\mathrm{k}}(\bar{x})$.

Then there exists one $\bar{\lambda} \in R^{p}, \bar{y} \in R^{m}$ such that $(\bar{x}, \bar{y}, \bar{\lambda})$ is efficient for (VD). Further if the assumptions of Theorem 2 are satisfied, then $(\bar{x}, \bar{y}, \bar{\lambda})$ is an efficient solution of (VD).

PROOF: Since $\bar{x}$ is efficient solution by Lemma 1, it is an optimal solution of $\mathrm{P}_{\mathrm{k}}(\bar{x})$. By Proposition 1, this implies that there exists $\lambda^{T}=\left(\lambda^{1}, \ldots, \lambda^{p}\right)$ and piecewise smooth $y: I \rightarrow R^{m}$ such that,

$$
\begin{gathered}
\bar{\lambda}_{k}\left(f_{x}^{k}-D f_{\dot{x}}^{k}+D^{2} f_{\dot{x}}^{k}\right)+\sum_{i \neq k} \bar{\lambda}_{i}\left(f_{x}^{i}-D f_{\dot{x}}^{i}+D^{2} f_{\ddot{x}}^{i}\right) \\
+\left(y(t)^{T} g_{x}-D y(t)^{T} g_{\dot{x}}+D^{2} y(t)^{T} g_{\ddot{x}}\right)=0 \\
\left(\lambda^{T} f_{x}+y(t)^{T} g_{x}\right)-D\left(\lambda^{T} f_{\dot{x}}+y(t)^{T} g_{\dot{x}}\right) \\
+D^{2}\left(\lambda^{T} f_{\ddot{x}}+y(t)^{T} g_{\ddot{x}}\right)=0, t \in I \\
\bar{y}(t)^{T} g(t, \bar{x}, \dot{\bar{x}}, \ddot{\bar{x}}) d t=0 \quad, t \in I \\
(\bar{\lambda}, \bar{y}(t)) \geq 0, t \in I \\
(\bar{\lambda}, \bar{y}(t)) \neq 0 \quad, \quad t \in I
\end{gathered}
$$

From (17), we have

$$
\int_{1} y(t)^{T} g(t, x, \dot{x}, \ddot{x}) d t=0
$$

Equations (16), (17) and (18) imply that $(\bar{x}, \bar{\lambda}, \bar{y})$ is feasible for (M-WD). The equality of objective functional of primal and dual problems is obvious from their formulations. Efficiency of $(\bar{x}, \bar{\lambda}, \bar{y})$ is immediate from the application of Theorem 2.

As in [4], by employing chain rule in calculus, it can be easily seen that the expression $\left(\lambda^{T} f_{x}+y(t)^{T} g_{x}\right)$ $D\left(\lambda^{T} f_{\dot{x}}+y(t)^{T} g_{\dot{x}}\right)+D^{2}\left(\lambda^{T} f_{\ddot{x}}+y(t)^{T} g_{\dot{x}}\right)$, may be regarded as a function $\theta$ of variables $t, x, \dot{x}, \ddot{x}, \dddot{x}, y, \dot{y}, \ddot{y}$ and $\lambda$, where $\dddot{x}=D^{3} x$ and $\ddot{y}=D^{2} y$. That is, we can write 


$$
\begin{aligned}
& \theta(t, x, \dot{x}, \ddot{x}, \dddot{x}, y, \dot{y}, \ddot{y}, \lambda)=\left(\lambda^{T} f_{x}+y(t)^{T} g_{x}\right) \\
& -D\left(\lambda^{T} f_{\dot{x}}+y(t)^{T} g_{\dot{x}}\right)+D^{2}\left(\lambda^{T} f_{\ddot{x}}+y(t)^{T} g_{\ddot{x}}\right)
\end{aligned}
$$

In order to prove converse duality between (VP) and (M-WD), the space $X$ is now replaced by a smaller space $X_{2}$ of piecewise smooth thrice differentiable function $x: I \rightarrow R^{n} \quad$ with the norm $\|x\|_{\infty}+\|D x\|_{\infty}+\left\|D^{2} x\right\|_{\infty}+$ $\left\|D^{3} x\right\|_{\infty}$. The problem (M-WD) may now be briefly written as, Minimize

$$
\left(-\int_{i} f^{1}(t, x, \dot{x}, \ddot{x}) d t, \ldots,-\int_{i} f^{p}(t, x, \dot{x}, \ddot{x}) d t\right)
$$

Subject to

$$
\begin{gathered}
x(a)=0=x(b) \\
\dot{x}(a)=0=\dot{x}(b) \\
\theta(t, x, \dot{x}, \ddot{x}, \dddot{x}, y, \dot{y}, \ddot{y}, \lambda)=0 \\
\int_{1} y(t)^{T} g(t, x, \dot{x}, \ddot{x}) d t \stackrel{\geq}{=} \\
y(t) \geq 0, t \in I
\end{gathered}
$$

Consider $\quad \theta(t, x(\cdot), \dot{x}(\cdot), \ddot{x}(\cdot), \dddot{x}(\cdot), y(\cdot), \dot{y}(\cdot), \ddot{y}(\cdot), \lambda)=0$ as defining a mapping $\psi: X_{2} \times Y \times R^{p} \rightarrow B$ where $Y$ is a space of piecewise twice differentiable function and $B$ is the Banach Space. In order to apply Theorem 1 to the problem $(\mathrm{M}-\mathrm{WD})$, the infinite dimensional inequality must be restricted. In the following theorem, we use $\psi^{\prime}$ to represent the Frèchèt derivative

$$
\left[\psi_{x}(x, y, \lambda), \psi_{y}(x, y, \lambda), \psi_{\lambda}(x, y, \lambda)\right] .
$$

THEOREM 4. (Converse Duality): Let $D$ be an efficient solution with $x \in X_{2}, \quad y \in Y_{2}$ and $\lambda^{T} \in R^{p}$ and $\psi^{\prime}$ have a (weak*) closed range hypothesis. Let $f$ and $g$ be twice continuously differentiable. Assume that

(H) $\int_{I} \lambda^{T} f d t$ be pseudoinvex and $\int_{I} y(t)^{T} g d t$ be quasi-invex with respect to same $\eta$.

$\left(\mathbf{H}_{2}\right)$

$$
\begin{aligned}
& \sigma(t)^{T}\left(\sigma(t) \theta_{x}-D \sigma(t) \theta_{\dot{x}}+D^{2} \sigma(t) \theta_{\ddot{x}}-D^{3} \sigma(t) \theta_{\ddot{x}}\right)=0 \\
& \quad \Rightarrow \sigma(t)=0, t \in I .
\end{aligned}
$$

(H) $f_{x}^{i}-D f_{\dot{x}}^{i}+D^{2} f_{\ddot{x}}^{i}, i=1,2, \ldots, p$ are linearly independent.

Then $\bar{x}$ is an efficient solution of (VP).

Proof: Since $(\bar{x}, \bar{\lambda}, \bar{y})$ where $\bar{x} \in X$ and $\psi^{\prime}$ having a closed range, is an efficient solution of (M-WD), by Theorem 2, it implies that there exist $\alpha \in R, \gamma \in R$, $\eta \in R^{p} \quad$ and piecewise smooth $\beta: R \rightarrow R^{n}$ and $\mu: R \rightarrow R^{m}$ satisfying the following conditions .

$$
\begin{gathered}
-\alpha\left(f_{x}-D f_{\dot{x}}+D^{2} f_{\ddot{x}}\right) \\
-\gamma\left(y(t)^{T} g_{x}-D\left(y(t)^{T} g_{\dot{x}}\right)+D^{2}\left(y(t)^{T} g_{\ddot{x}}\right)\right) \\
+\beta(t)^{T} \theta_{x}-D\left(\beta(t)^{T} \theta_{\dot{x}}\right)+D^{2}\left(\beta(t)^{T} \theta_{\ddot{x}}\right) \\
-D^{3}\left(\beta(t)^{T} \theta_{\ddot{x}}\right)=0
\end{gathered}
$$

$$
\beta(t)^{T} \theta_{y}-D\left(\beta(t)^{T} \theta_{\dot{y}}\right)+D^{2}\left(\beta(t)^{T} \theta_{\ddot{y}}\right)-\gamma g-\mu(t)=0
$$

$$
\begin{gathered}
\beta(t)^{T}\left(f_{x}-D f_{\dot{x}}+D^{2} f_{\ddot{x}}\right)-\eta=0 \\
\gamma \int_{1} y(t)^{T} g(t, x, \dot{x}, \ddot{x}) d t=0 \\
\eta^{T} \lambda=0, \quad \mu(t)^{T} y(t)=0, \quad t \in I \\
(\alpha, \gamma, \eta, \mu(t)) \geq 0, \quad t \in I \quad \text { and } \\
(\alpha, \gamma, \eta, \mu(t), \beta(t)) \neq 0, \quad t \in I
\end{gathered}
$$

Since $\lambda>0, \quad \eta^{T} \lambda=0$, which implies $\eta=0$ This yields from (23)

$$
\beta(t)^{T}\left(f_{x}-D f_{\dot{x}}+D^{2} f_{\ddot{x}}\right)=0
$$

Using the equality constraint (11) in (21), we have

$$
\begin{gathered}
-(\alpha-\gamma \lambda)^{T}\left(f_{x}-D f_{\dot{x}}+D^{2} f_{\ddot{x}}\right)+\beta(t)^{T} \theta_{x} \\
-D\left(\beta(t)^{T} \theta_{\dot{x}}\right)+D^{2}\left(\beta(t)^{T} \theta_{\ddot{x}}\right)-D^{3}\left(\beta(t)^{T} \theta_{\ddot{x}}\right)=0
\end{gathered}
$$

Postmultiplying Equation (21) by $\beta(t)$ and using (27) in (28) we get,

$$
\beta(t)^{T}\left(\beta(t)^{T} \theta_{\dot{x}}\right)+D^{2}\left(\beta(t)^{T} \theta_{\ddot{x}}\right)-D^{3}\left(\beta(t)^{T} \theta_{\ddot{x}}\right)=0, t \in I
$$

This by hypothesis $\left(\mathrm{H}_{2}\right)$ implies $\beta(t)=0, t \in I$

Also from (28) we have

$$
(\alpha-\gamma \lambda)^{T}\left(f_{x}-D f_{\dot{x}}+D^{2} f_{\ddot{x}}\right)=0
$$

This, because of linear independence of $f_{x}^{i}-D f_{\dot{x}}^{i}+$ $D^{2} f_{\ddot{x}}^{i}, i=1,2, \ldots, p$, gives

$$
\alpha-\gamma \lambda=0
$$

Now suppose $\gamma=0$, then, from (22) and (29) we have $\mu(t)=0, t \in I$ and $\alpha=0$ respectively.

This implies $(\alpha, \beta(t), \gamma, \eta, \mu(t))=0$, which is the 
contradiction to $(\alpha, \beta(t), \gamma, \eta, \mu(t)) \neq 0, t \in I$.

Hence $\gamma>0$ and by (29) we have, $\alpha>0$.

The relation (22) in conjunction with $\beta(t)=0$, and $\mu(t) \geq 0, t \in I$ gives

$$
g(t, x, \dot{x}, \ddot{x}) \leqq 0 \quad, t \in I
$$

This implies the feasibility of $\bar{x}$ for (VP) and its efficiency is evident from and application of Theorem 2.

\section{Natural Boundary Values}

The duality results obtained in the preceding sections can easily be extended to the multiobjective variational problems with natural boundary values rather than fixed end points.

Primal $\left(\mathbf{P}_{1}\right)$ Minimize

$$
\left(\int_{I} f^{1}(t, x, \dot{x}, \ddot{x}) d t, \ldots, \int_{I} f^{p}(t, x, \dot{x}, \ddot{x}) d t\right)
$$

Subject to

$$
g(t, x, \dot{x}, \ddot{x}) \leq 0 \quad, \quad t \in I
$$

Dual $\left(\mathbf{D}_{1}\right)$ Maximize

$$
\left(\int_{I} f^{1}(t, x, \dot{x}, \ddot{x}) d t, \ldots, \int_{I} f^{p}(t, x, \dot{x}, \ddot{x}) d t\right)
$$

Subject to

$$
\begin{gathered}
\left(\lambda^{T} f_{x}+y(t)^{T} g_{x}\right)-D\left(\lambda^{T} f_{\dot{x}}+y(t)^{T} g_{\dot{x}}\right) \\
+D^{2}\left(\lambda^{T} f_{\ddot{x}}+y(t)^{T} g_{\ddot{x}}\right)=0 \quad t \in I, \\
y(t)^{T} g_{\dot{x}}=0, a t \quad t=a \text { and } t=b, \\
y(t)^{T} g_{\ddot{x}}=0, a t \quad t=a \text { and } t=b, \\
y(t) \geq 0, t \in I .
\end{gathered}
$$

\section{Nonlinear Programming}

If the problems $\left(\mathrm{P}_{1}\right)$ and $\left(\mathrm{D}_{1}\right)$ are independent of $t$, then they will reduce to the following multiobjective nonlinear programming problems studied in [15]

(NP): Minimize $f(x)$

Subject to

$$
g(x) \leq 0 .
$$

(ND): Maximize $f(x)$

Subject to

$$
\begin{gathered}
\lambda^{T} f_{x}+y^{T} g_{x}=0 \\
\lambda>0, \quad y \geqq 0 .
\end{gathered}
$$

\section{References}

[1] R. Courant and D. Hilbert, "Methods of Mathematical Physics," Wiley, New York, Vol. 1, 1943.

[2] K. O. Friedrichs, "Ein Verfrahren der Variations-Rechung das Minimum eines Integrals Maximum eines Anderen Ausdruckes Dazustellan,” Göttingen Nachrichten, 1929.

[3] M. A. Hanson, "Bonds for Functionally Convex Optimal Control Problems," Journal of Mathematical Analysis and Applications, Vol. 8, No. 1, February 1964, pp. 84-89.

[4] B. Mond and M. A. Hanson, "Duality for Variational Problems," Journal of Mathematical Analysis and Applications, Vol. 18, No. 2, May 1967, pp. 355-364.

[5] F. A. Valentine, "The Problem of Lagrange with Differential Inequalities as Added Side Conditions," Contributions to the Calculus of Variations, 1933-1937, University of Chicago Press, 1937, pp. 407-448.

[6] C. R. Bector, S. Chandra and I. Husain, "Generalized Concavity and Duality in Continuous Programming," Utilitas Mathematica, Vol. 25, 1984, pp. 171-190.

[7] S. Chandra, B. D. Craven and I. Husain, "A Class of Nondifferentiable Continuous Programming Problems," Journal of Mathematical Analysis Applications, Vol. 107, No. 1, April 1985, pp. 122-131.

[8] S. Chandra, B. D. Craven and I. Husain, "Continuous Programming Containing Arbitrary Norms," Journal of Australian Mathematical Society (Series A), Vol. 39, No. 1, 1985, pp. 28-38.

[9] I. Husain and Z. Jabeen, "On Variational Problems Involving Higher Order Derivatives," Journal of Applied Mathematics and Computing, Vol. 27, No. 1-2, March 2005, pp. 433-455.

[10] B. Mond and S. Chandra and I. Husain, "Duality of Variational Problems with Invexity," Journal of Mathematical Analysis and Applications, Vol. 134, No. 2, September 1988, pp. 322-328.

[11] C. R. Bector and I. H. Husain, "Duality for Multiobjective Variational Problems," Journal of Mathematical Analysis and Applications, Vol. 166, No. 1, 1 May 1992, pp. 214-224.

[12] X. H. Chen, "Duality for Multiobjective Variational Problems with Invexity" Journal of Mathematical Analysis and Appliactions, Vol. 203, No. 1, October 1996, pp. 236-253.

[13] B. Mond and I. Smart, "Duality with Invexity for a Class of Nondifferentiable Static and Continuous Programming Problems," Journal of Mathematical Analysis and Applications, Vol. 136, 1988, pp. 325-333.

[14] V. Chankong and Y. Y. Haimes, "Multiobjective Decision Making: Theory and Methodology," North Holland, New York, 1983.

[15] R. R. Egudo and M. A. Hanson, "Multiobjective Duality with Invexity," Journal of Mathematical Analysis and Appliactions, Vol. 126, No. 2, September 1987, pp. 469- 477. 